\title{
OPEN Effect of endometrial preparation protocols on the risk of ectopic pregnancy for frozen embryo transfer
}

\author{
Seung Chik Jwa ${ }^{1 \bowtie}$, Masashi Takamura ${ }^{1}$, Akira Kuwahara ${ }^{2}$, Takeshi Kajihara $^{1}$ \& \\ Osamu Ishihara ${ }^{1}$
}

Studies have consistently reported a significantly reduced incidence of ectopic pregnancy (EP) for frozen-thawed embryo transfer (ET) cycles compared with fresh cycles. However, only a few studies reported an association between endometrial preparation protocols on EP and results were conflicting. A registry-based retrospective cohort study of 153,354 clinical pregnancies following frozen single ETs between 2014 and 2017 were conducted, of which 792 cases of EP (0.52\%) were reported. Blastocyst embryo transfers accounted for $87 \%$ of the total sample and were significantly associated with a decreased risk for EP compared with early cleavage ET $(0.90 \%$ vs. $0.46 \%$, adjusted $\mathrm{OR}=0.50,95 \% \mathrm{Cl}, 0.41$ to 0.60$)$. Compared with natural cycles, hormone replacement cycles (HRC) demonstrated a similar risk for EP $(0.53 \%$ vs. $0.47 \%$, adjusted $\mathrm{OR}=1.12,95 \% \mathrm{Cl}, 0.89$ to 1.42). Subgroup analysis with or without tubal factor infertility and early cleavage/blastocyst ETs demonstrated similar non-significant associations. Endometrial preparation protocols using clomiphene (CC) were associated with a significantly increased risk for EP (1.12\%, adjusted OR $=2.34$; $95 \% \mathrm{Cl}, 1.38$ to 3.98). These findings suggest that HRC and natural cycles had a similar risk for EP. Endometrial preparation using CC was associated with an increased risk of EP in frozen embryo transfer cycles.

Despite significant improvements in in vitro fertilization (IVF) techniques, ectopic pregnancy (EP) remains a serious problem in assisted reproductive technology (ART), occurring $2-5 \%{ }^{1-3}$ higher than in the general population $(1-2 \%)^{4-6}$. Various factors in ART are associated with EP, such as the number of embryos transferred ${ }^{7}$, assisted hatching ${ }^{8}$ and embryo stage at embryo transfer $(E T)^{9}$. Further, patient related factors such as tubal factor infertility diagnosis, endometriosis, and smoking are also associated with the risk of $\mathrm{EP}^{10-13}$. Studies have consistently reported a significantly reduced incidence of EP for frozen-thawed ET cycles compared with fresh cycles $^{7,14-16}$. The lower risk of EP for frozen-thawed ET cycles may reflect the more physiological hormonal environment compared with stimulated cycles $^{1,17}$.

Several endometrial preparation protocols, such as natural ovulatory cycle, hormone replacement cycles (HRC) and ovarian stimulation using exogeneous gonadotropin ( $\mathrm{Gn}$ ), clomiphene (CC) and letrozole, have been applied in frozen-thawed ET cycles. Although these cycles were more equivalent to the physiological hormonal environment than fresh cycles, because HRC and ovarian stimulation cycles use medication, the hormonal environments are different among different endometrial preparation protocols.

To date, only a few studies reported an association between endometrial preparation protocols on EP and results were conflicting ${ }^{12,18,19}$. In China, recent studies investigating the effect of endometrial thickness on EP in frozen-thawed ET cycles reported that the EP rate for HRC cycles was more than double the rate for natural cycles $(4.7 \% \text { vs. } 2.1 \% \text {, respectively })^{12}$. However, double embryo transfer and early cleavage stage ET in that study accounted for $86 \%$ and $84.9 \%$ of the cases examined, respectively, both of which could affect the incidence of EP. The differences in ART practice and extremely high EP rate (3.15\%) limit the generalization of their study results $^{20}$.

In Japan, single embryo transfer (SET) has accounted for more than $80 \%$ of ART cases because of the recommendation by the Japan Society of Obstetrics and Gynecology (JSOG) in $2008^{21}$. Furthermore, the mandatory

\footnotetext{
${ }^{1}$ Department of Obstetrics and Gynecology, Saitama Medical University, 38 Morohongo, Moroyama, Saitama 350-0495, Japan. '2Department of Obstetrics and Gynecology, Graduate School of Biomedical Sciences, Tokushima University, Tokushima, Japan. ${ }^{凶}$ email: jwa_s@saitama-med.ac.jp
} 
reporting system of the Japanese ART registry is a cycle-based format, with almost all treatment cycles registered. Since 2014, endometrial preparation protocols using HRC were collected in this registry. The current study aimed to evaluate the risk of EPs for frozen-thawed single ET cycles on the basis of different endometrial preparation protocols.

\section{Results}

Baseline characteristics of the sample population stratified by EP are shown in Table 1. Among 153,354 clinical pregnancies and 792 cases of EP (0.52\%) were reported. Mean maternal age was 36 years, which was similar in the EP and non-EP groups. EP was significantly more frequent in patients exhibiting tubal factor infertility $(0.95 \%)$ compared with cases without tubal factor infertility $(0.44 \%)(P<0.001)$. Similarly, endometriosis was significantly associated with an increased incidence of EP, while infertile couples with male factor and unexplained infertility had a significantly lower incidence of EP compared with infertile couples without these diagnoses. Among endometrial preparation protocols, the EP rate was highest in cycles using CC (1.12\%), followed by HRC $(0.53 \%)$, and the lowest rate was in the natural cycle $(0.47 \%)$ group; with overall analysis showing a significant association between endometrial preparation protocols and EP $(P=0.004)$. The EP rate was significantly higher for early cleavage stage ET $(0.90 \%)$ than blastocyst ET $(0.46 \%)(P<0.001)$.

Associations between baseline characteristics and endometrial preparation protocols are shown in Supplemental Table 1. Maternal age was highest in the natural cycle group (mean $=36.8$, standard deviation $[\mathrm{SD}]=3.9)$, while the HRC $($ mean $=35.4, \mathrm{SD}=4.1)$ and $\mathrm{CC}($ mean $=35.4, \mathrm{SD}=4.1)$ groups had the youngest ages. The HRC and letrozole groups had the highest prevalence of polycystic ovarian syndrome (PCOS) $(5.5 \%$ and $8.7 \%$, respectively). Blastocyst stage ET was performed in more than $85 \%$ of patients in the natural, HRC, letrozole and Gn groups. Early cleavage stage ET was most frequent in the CC group (26.6\%).

Crude and adjusted ORs for EP after various ART treatments are shown in Table 2. The crude and adjusted ORs for HRC (adjusted OR =1.12, 95\% CI, 0.89 to 1.42) were not significantly different compared with the natural cycle. However, ovarian stimulation using CC demonstrated a significant higher adjusted OR (adjusted OR $=2.34$, 95\% CI, 1.38 to 3.98) for EP compared with the natural cycle. Blastocyst transfer was significantly associated with a decreased risk for EP (adjusted $\mathrm{OR}=0.50,95 \% \mathrm{CI}, 0.41$ to 0.60 ) compared with early cleavage ET.

Results of subgroup analysis for patients with or without tubal factor infertility, excluding both tubal factor and endometriosis, and early cleavage and blastocyst ETs are shown in Table 3. For the subgroups of tubal factor infertility $(n=23,114)$ and early cleavage ETs $(n=19,731)$, there were no significant associations between endometrial preparation protocols and the risk of EP. Conversely, for subgroups without tubal factor infertility diagnosis $(n=130,340$ cycles), excluding tubal factor and endometriosis $(n=121,341$ cycles $)$ and blastocyst ET cycles ( $n=133,479$ cycles), significant associations were found for endometrial preparations using CC and the risk of EP; without tubal infertility $(\mathrm{OR}=2.31,95 \% \mathrm{CI}, 1.33$ to 4.01$)$, excluding tubal factor and endometriosis $(\mathrm{OR}=2.14,95 \% \mathrm{CI}, 1.18$ to 3.86$)$ and with blastocyst ET $(\mathrm{OR}=2.66,95 \% \mathrm{CI}, 1.50$ to 4.72$)$, compared with natural cycles.

\section{Discussion}

This study analyzed a large nationally-representative ART register for frozen-thawed single ETs in Japan, and found an EP rate of $0.52 \%$, which was much smaller than the recently reported EP rate for fresh single ET cycles $(1.46 \%)$ using the same Japanese registry ${ }^{22}$. Single ET using blastocysts resulted in an almost $50 \%$ reduction in the risk of EP compared with early cleavage stage ET. There was no significant difference between natural cycles and HRC for the risk of EP. The use of CC during endometrial preparation was associated with a significantly increased risk for EP. To date, this is the largest study reporting the effect of endometrial preparation protocols on the risk of EP in frozen single ET cycles.

The lower risk for EP in frozen-thawed blastocyst ET cycles compared with fresh ET cycles was first reported by Ishihara et al. using the Japanese ART registry ${ }^{16}$. Since then, several studies have reported a similar risk reduction for EP in frozen compared with fresh ET cycles ${ }^{14,23}$. Recent meta-analysis investigated the risks of EP in frozen-thawed versus fresh blastocyst ETs using fourteen retrospective studies $(\mathrm{n}=251,762$ cycles) and demonstrated that the EP rate for fresh single blastocyst ET cycles $(1.2 \%)$ was significantly higher than that for frozen-thawed blastocyst ET cycles $(0.80 \%)^{23}$. One of the differences between fresh and frozen cycles is the ovarian stimulation. Our previous study of the effect of ovarian stimulation protocols on the risk of EP demonstrated that the EP rate for natural (i.e., unstimulated) IVF cycles was $0.47 \%$, significantly lower than the rate for stimulated cycles (1.47-2.18\%). Interestingly, the EP rate for unstimulated fresh cycles $(0.47 \%)$ was similar to the EP rate for all frozen-thawed cycles $(0.52 \%)$ examined in the current study. These results indicate that ovarian stimulation may mediate the elevated risk of EP for the fresh cycles.

For the frozen-thawed cycles in our study, the risk of EP for blastocyst ET was almost halved compared with early cleavage stage ET, consistent with previous studies. Recent meta-analysis of 22 studies (143,643 pregnancies) investigated the effect of embryo stage at transfer on EP and found that the risk of EP was significantly lower for blastocyst ET than early cleavage stage ET for both fresh (relative risk $[\mathrm{RR}]=0.78,95 \% \mathrm{CI}, 0.69$ to 0.88 ) and frozen-thawed ETs $(\mathrm{RR}=0.43,95 \% \mathrm{CI}, 0.36 \text { to } 0.51)^{9}$. It was suggested that the time period for implantation would be reduced for blastocysts compared with early cleavage stage embryos after ET, and would therefore provide a lower chance to migrate to fallopian tubes ${ }^{24}$. Furthermore, uterine contraction 3-5 days after ovulation would direct embryos from the uterine cervix towards the fundus, so early cleavage stage embryos would be more likely to migrate to the fallopian tubes ${ }^{25}$. Another potential explanation for the decreased risk of EP for blastocyst embryos maybe the larger size of blastocysts compared with cleavage stage embryos, preventing migration to fallopian tubes ${ }^{26}$. 


\begin{tabular}{|c|c|c|c|}
\hline Characteristics & Non-ectopic pregnancy & Ectopic pregnancy & $P$ value \\
\hline Maternal age, (year) & $36.0(4.1)$ & $36.1(4.0)$ & 0.28 \\
\hline$<30$ & $10,156(99.6)$ & $46(0.45)$ & \multirow{4}{*}{0.71} \\
\hline $30-34$ & $44,455(99.5)$ & $229(0.51)$ & \\
\hline $35-39$ & $65,840(99.5)$ & $354(0.53)$ & \\
\hline$\geq 40$ & $32,111(99.5)$ & $163(0.51)$ & \\
\hline \multicolumn{4}{|l|}{ Tubal factor infertility } \\
\hline Yes & $22,894(99.1)$ & $220(0.95)$ & \multirow{2}{*}{$<0.001$} \\
\hline No & $129,668(99.6)$ & $572(0.44)$ & \\
\hline \multicolumn{4}{|l|}{ Endometriosis } \\
\hline Yes & $10,576(99.3)$ & $80(0.75)$ & \multirow{2}{*}{$<0.001$} \\
\hline No & $141,986(99.5)$ & $712(0.50)$ & \\
\hline \multicolumn{4}{|l|}{ Antisperm antibody } \\
\hline Yes & $772(99.1)$ & $7(0.90)$ & \multirow{2}{*}{0.14} \\
\hline No & $151,790(99.5)$ & $785(0.51)$ & \\
\hline \multicolumn{4}{|l|}{ Male factor } \\
\hline Yes & $42,900(99.6)$ & $177(0.41)$ & \multirow{2}{*}{$<0.001$} \\
\hline No & $109,662(99.4)$ & $615(0.56)$ & \\
\hline \multicolumn{4}{|l|}{ PCOS/anovulation } \\
\hline Yes & 6515 (99.6) & $29(0.44)$ & \multirow{2}{*}{0.40} \\
\hline No & $146,047(99.5)$ & $763(0.52)$ & \\
\hline \multicolumn{4}{|l|}{ Others } \\
\hline Yes & $20,772(99.4)$ & $127(0.61)$ & \multirow{2}{*}{0.048} \\
\hline No & $131,790(99.5)$ & $665(0.50)$ & \\
\hline \multicolumn{4}{|l|}{ Unexplained } \\
\hline Yes & $67,027(99.6)$ & $283(0.42)$ & \multirow{2}{*}{$<0.001$} \\
\hline No & $85,535(99.4)$ & $509(0.59)$ & \\
\hline \multicolumn{4}{|c|}{ Endometrial preparation protocol } \\
\hline Natural & $45,281(99.5)$ & $216(0.47)$ & \multirow{5}{*}{0.004} \\
\hline HRC & $100,275(99.5)$ & $536(0.53)$ & \\
\hline Clomiphene & $1586(98.9)$ & $18(1.12)$ & \\
\hline Letrozole & $4246(99.6)$ & $16(0.38)$ & \\
\hline Gonadotropin & $1174(99.5)$ & $6(0.51)$ & \\
\hline \multicolumn{4}{|l|}{ Embryo stage at transfer } \\
\hline Early cleavage & $19,697(99.1)$ & $178(0.90)$ & \multirow{2}{*}{$<0.001$} \\
\hline Blastocyst & $132,865(99.5)$ & $614(0.46)$ & \\
\hline \multicolumn{4}{|l|}{ Assisted hatching ${ }^{b}$} \\
\hline$(+)$ & $104,201(99.5)$ & $525(0.50)$ & \multirow{2}{*}{0.22} \\
\hline$(-)$ & $48,358(99.5)$ & $267(0.55)$ & \\
\hline \multicolumn{4}{|l|}{ Luteal support $^{\mathrm{c}}$} \\
\hline None & $8576(99.6)$ & $38(0.44)$ & 0.32 \\
\hline Progesterone & $36,916(99.6)$ & $156(0.42)$ & 0.003 \\
\hline $\mathrm{hCG}$ & $6184(99.4)$ & $38(0.61)$ & 0.29 \\
\hline Progesterone +hCG & 7297 (99.4) & $48(0.65)$ & 0.09 \\
\hline Estrogen + Progesterone & $93,761(99.5)$ & $518(0.55)$ & 0.02 \\
\hline Others & 3709 (99.5) & $20(0.54)$ & 0.86 \\
\hline Year & & & \\
\hline 2014 & $29,725(99.5)$ & $163(0.55)$ & \\
\hline 2015 & $35,716(99.5)$ & $194(0.54)$ & 106 \\
\hline 2016 & $41,340(99.5)$ & $202(0.49)$ & 0.64 \\
\hline 2017 & $45,781(99.5)$ & $233(0.51)$ & \\
\hline
\end{tabular}

Table 1. Baseline characteristics of the sample population stratified by ectopic pregnancies. $(\mathrm{n}=153,354)$. Data are presented as mean (SD) for continuous variables and n (\%) for dichotomous variables. Percentages are presented in the rows for the purpose of comparison. $h C G$ human chorionic gonadotropin, HRC hormone replacement cycle, $P C O S$ polycystic ovarian syndrome. ${ }^{a} P$ values were assessed with the use of the $\chi^{2}$ or Student's t test. ${ }^{\mathrm{b}} 3$ cases were missing in the variable. ${ }^{\mathrm{c}}$ Multiple answers were allowed. 


\begin{tabular}{|l|l|l|}
\hline \multicolumn{2}{|l|}{ Crude OR (95\% CI) } & Adjusted OR (95\% CI) \\
\hline Endometrial preparation protocol & Reference & Reference \\
\hline Natural & $1.12(0.89$ to 1.41$)$ & $1.12(0.89$ to 1.42$)$ \\
\hline HRC & $\mathbf{2 . 3 8}(1.41$ to 4.01$)$ & $\mathbf{2 . 3 4}(1.38$ to 3.98) \\
\hline Clomiphene & $0.79(0.49$ to 1.29$)$ & $0.81(0.49$ to 1.32$)$ \\
\hline Letrozole & $1.07(0.43$ to 2.67$)$ & $1.02(0.41$ to 2.54$)$ \\
\hline Gonadotropin & \multicolumn{2}{|l}{} \\
\hline Embryo stage at transfer & Reference & Reference \\
\hline Early cleavage & $\mathbf{0 . 5 1}(\mathbf{0 . 4 3}$ to $\mathbf{0 . 6 1})$ & $\mathbf{0 . 5 0}(\mathbf{0 . 4 1}$ to 0.60) \\
\hline Blastocyst & \multicolumn{2}{|l}{} \\
\hline Assisted hatching & Reference & Reference \\
\hline$(-)$ & $1.10(0.89$ to 1.35$)$ & $1.08(0.88$ to 1.33$)$ \\
\hline$(+)$ & \multicolumn{2}{|l}{} \\
\hline
\end{tabular}

Table 2. Risk of ectopic pregnancy for ART treatment using frozen single embryo transfer cycles $(\mathrm{n}=153,354)$. Significantly increased or reduced odds are indicated by boldface. $C I$ confidence interval, $H R C$ hormone replacement cycle, $O R$ odds ratio. ${ }^{a}$ Adjusted for maternal age, infertility diagnosis of tubal factor, endometriosis, male factor, other or unexplained and year.

\begin{tabular}{|c|c|c|}
\hline & Crude OR $(95 \% \mathrm{Cl})$ & Adjusted OR (95\% Cl) \\
\hline \multicolumn{3}{|c|}{ Tubal factor infertility $(n=23,114)^{a}$} \\
\hline Natural & Reference & Reference \\
\hline HRC & $1.32(0.93$ to 1.87$)$ & $1.37(0.95$ to 1.98$)$ \\
\hline Clomiphene & $2.35(0.81$ to 6.72$)$ & $2.29(0.80$ to 6.55$)$ \\
\hline Letrozole & $0.86(0.38$ to 1.93$)$ & $0.86(0.38$ to 1.93$)$ \\
\hline Gonadotropin & $0.57(0.09$ to 3.73$)$ & $0.59(0.09$ to 3.82$)$ \\
\hline \multicolumn{3}{|c|}{ Without tubal factor infertility $(n=130,240)^{a}$} \\
\hline Natural & Reference & Reference \\
\hline HRC & $1.05(0.83$ to 1.34$)$ & $1.03(0.82$ to 1.30$)$ \\
\hline Clomiphene & $2.42(1.42$ to 4.14$)$ & $2.31(1.33$ to 4.01$)$ \\
\hline Letrozole & $0.78(0.48$ to 1.26$)$ & $0.79(0.49$ to 1.29$)$ \\
\hline Gonadotropin & $1.25(0.47$ to 3.31$)$ & $1.22(0.46$ to 3.23$)$ \\
\hline \multicolumn{3}{|c|}{ Without tubal factor and endometriosis $(n=121,341)^{b}$} \\
\hline Natural & Reference & Reference \\
\hline HRC & $1.002(0.79$ to 1.27$)$ & $1.006(0.79$ to 1.28$)$ \\
\hline Clomiphene & $2.22(1.26$ to 3.93$)$ & $2.14(1.18$ to 3.86$)$ \\
\hline Letrozole & $0.73(0.46$ to 1.17$)$ & $0.73(0.46$ to 1.18$)$ \\
\hline Gonadotropin & $0.80(0.24$ to 2.65$)$ & $0.79(0.24$ to 2.65$)$ \\
\hline \multicolumn{3}{|c|}{ Early cleavage $(n=19,731)^{c}$} \\
\hline Natural & Reference & Reference \\
\hline HRC & $0.75(0.49$ to 1.15$)$ & $0.74(0.49$ to 1.11$)$ \\
\hline Clomiphene & $1.07(0.41$ to 2.78$)$ & $1.13(0.42$ to 3.03$)$ \\
\hline Letrozole & $0.98(0.45$ to 2.17$)$ & $0.99(0.45$ to 2.17$)$ \\
\hline Gonadotropin & NA & NA \\
\hline \multicolumn{3}{|c|}{ Blastocyst $(n=133,479)^{c}$} \\
\hline Natural & Reference & Reference \\
\hline HRC & $1.20(0.98$ to 1.46$)$ & $1.20(0.98$ to 1.47$)$ \\
\hline Clomiphene & $2.75(1.58$ to 4.80$)$ & $2.66(1.50$ to 4.72$)$ \\
\hline Letrozole & $0.76(0.43$ to 1.36$)$ & $0.78(0.43$ to 1.40$)$ \\
\hline Gonadotropin & $1.44(0.56$ to 3.71$)$ & $1.38(0.53$ to 3.54$)$ \\
\hline
\end{tabular}

Table 3. Subgroup analysis of crude and adjusted ORs for ectopic pregnancy with endometrial preparation protocols. Significantly increased or reduced odds are indicated by boldface. $C I$ confidence interval, $H R C$ hormone replacement cycle, $N A$ not available, $O R$ odds ratio. ${ }^{a}$ Adjusted for maternal age, infertility diagnosis of endometriosis, male factor, other or unexplained and year. ${ }^{b}$ Adjusted for maternal age, infertility diagnosis of male factor, other or unexplained and year. ${ }^{c}$ Adjusted for maternal age, infertility diagnosis of tubal factor, endometriosis, male factor, other or unexplained and year. 
Although many studies have investigated endometrial preparation protocols and pregnancy and live birth rates $^{27-29}$, only a limited number of studies with conflicting results have investigated endometrial preparation protocols and the risk of $\mathrm{EP}^{12,18,19}$. Importantly, these studies did not specifically investigate endometrial preparation protocols and targeted other factors. One recent study investigated the effect of endometrial thickness on EP, using 17,244 pregnancies following frozen ETs from a single institution in China ${ }^{12}$. The study demonstrated that HRC was significantly associated with EP compared with the modified natural cycle (202/4294 [4.7\%] in HRC cycles vs. 105/4941 [2.1\%] in modified natural cycles, adjusted OR=2.25, 95\% CI, 1.76 to 2.87). However, double embryo transfer was performed in $86 \%$ of the total cycles, and early cleavage ETs predominated (84.9\%). Because of these characteristics, the EP rate was very high (3.15\%) in this Chinese sample, so caution is required with the reported association of HRC with EP. However, another Chinese study investigating the association between body mass index and EP using retrospective data $(n=16,378$ pregnancies $)$ demonstrated no significant associations between endometrial preparation protocols and the risk of EP for frozen cycles ${ }^{18}$. Furthermore, a retrospective analysis of clinical pregnancies in Italy following single blastocyst ETs with different endometrial preparation protocols demonstrated a significantly higher EP rate for natural cycles $(6 / 561,3.28 \%)$ than HRC $(4 / 585,1.83 \%)$ and modified natural cycles $(3 / 1749,0.4 \%)^{19}$. Our study, using the largest sample of subjects with single frozenthawed ET cycles demonstrated that the risk of EP did not differ for HRC compared with natural cycles.

Nevertheless, patient-related factors such as infertility diagnosis would still impact on the incidence of EP in frozen-thawed ET cycles. Boynukalin et al. investigated the risk of EP in 13,261 fresh and frozen-thawed ET cycles without tubal infertility cases and found that the risk of EP was the lowest in frozen-thawed ET cycles with embryos from previous freeze-all cycles (eFET, 0.9\%) than fresh ET cycles (1.6\%) and frozen-thawed ET with surplus embryos from a previous fresh cycle $(1 \%)^{30}$. Interestingly, day 3 ET in eFET group was a significantly lower risk for EP than day 5 ET $(1 / 360$ [0.03\%] vs. $15 / 1357$ [1.1\%], $P<0.05)$. Thus, we have conducted an additional analysis using the subgroup excluding tubal factor infertility diagnosis and endometriosis and blastocyst ET demonstrated still a significantly decreased risk for $\mathrm{EP}$ (adjusted $\mathrm{OR}=0.60,95 \% \mathrm{CI}, 0.49$ to 0.75 ). Thus, patientrelated factors and previous freeze-all in fresh cycles might impact on the risk of EP in frozen-thawed ET cycles.

The use of CC was associated with an increased risk of EP. Interestingly, our previous study investigating the effect of ovarian stimulation on the risk of EP for fresh cycles also demonstrated a significantly elevated risk of EP for ovarian stimulation using CC; EP rate of cycles using CC was the highest (1.81-2.18\%) compared with other ovarian stimulation regimens $(0.47-1.57 \%)^{22}$. One potential reason for the elevated risk of EP from cycles using CC was endometrial thickness. It is well known that ovulation induction using CC often results in a thinner endometrium, and it was reported that endometrial thickness in the mid-luteal phase was significantly thinner in women receiving $\mathrm{CC}$ compared with control cycles without $\mathrm{CC}^{31}$. Thinner endometrium during frozenthawed ET cycles was reported to be associated with an increased risk for $\mathrm{EP}^{12}$. Furthermore, the effect of CC on endometrial receptivity ${ }^{32}$ and apoptotic changes in fallopian tube epithelial cells have been reported ${ }^{33}$. Such changes in cycles using CC may result in reduced probability of embryo implantation in the uterine endometrium compared with the probability of EP per clinical pregnancy. Clinical pregnancy rates for frozen single ETs in the Japanese registry were much lower for CC cycles (27.0\%) than natural (37.0\%), HRC (34.3\%), letrozole (42.3\%) and $\mathrm{Gn}(32.9 \%)$ protocols (data not shown).

One strength of the current study was the large number of frozen-thawed single ET cycles, enabling the investigation of relatively rare but serious events of EP on the basis of various endometrial protocols. Because the Japanese ART registry system involves a mandatory registration system in conjunction with governmental subsidies, all treatment cycles are registered in the system, so sampling bias would be less likely. Furthermore, with the high single ET rate (more than $80 \%$ of ETs), the current study provides an EP rate using autologous embryos and eliminates the effect of number of embryos transferred. However, several limitations also exist in the study. The study was retrospective, so the medical indication for selecting endometrial preparation protocols was unknown. Thus, although we have adjusted for factors such as age and several infertility diagnosis, which were associated with endometrial preparation protocols (Supplemental Table 1), unmeasured confounders such as smoking, body mass index and ovarian reserve may impact the results. Furthermore, the current study lacked information on endometrial thickness, and estrogen and progesterone levels during the cycles, so we were unable to elucidate the possible mediating factors between endometrial preparation protocols and the risk of EP, especially in cycles using CC. Lastly, because $97.8 \%$ of the population in Japan is currently Japanese ${ }^{34}$, we cannot consider the effects of race and ethnicity. Similarly, advanced age is the major contributor for the increasing number of ART patients in Japan, and the mean maternal age of the current study was relatively high ( 36 years). Thus, to confirm the present findings, it is essential that prospective studies include potential confounders and mediators in different populations.

In conclusion, using the nationally representative ART data for a large Japanese sample with a high single ET rate, our study demonstrated that endometrial preparation protocols using HRC had a similar risk for EP compared with natural cycles. Given the increasing number of frozen-thawed ETs worldwide, the current results are reassuring for the use of HRC in terms of the risks of EP. Further research would be necessary to elucidate potential mediating factors, especially for the use of CC for endometrial preparation and the risk of EP.

\section{Materials and methods}

Sample selection. This was a registry-based retrospective cohort study using the Japanese national ART registry managed by the JSOG. The ART facilities in Japan have to register their treatment cycles for patients to receive government subsidies. The registry consists of cycle-specific information, including patient background and treatment information. Because treatment using donated oocytes is not allowed in Japan, all ET cycles are autologous. Furthermore, preimplantation genetic testing for chromosomal aneuploidy was not practiced in Japan during the study period. This study was approved by the institutional review board of Saitama Medical 


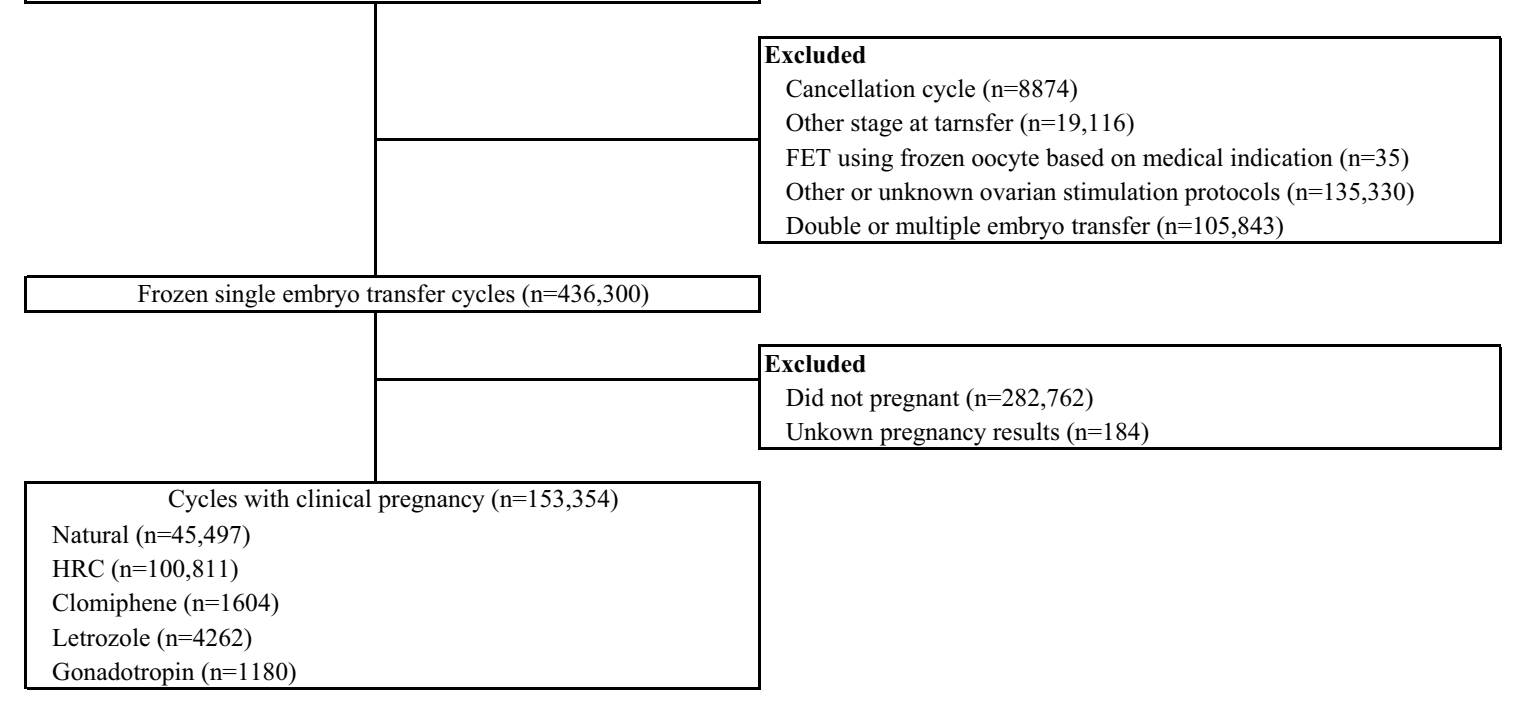

Figure 1. Flow diagram of cohort selection.

University (Approval number 916, April. 2020) and the ethics committee of the JSOG (Approval number 20206, September. 2020). The study was conducted in accordance with Japanese law and the STROBE Guidelines. Informed consent was waived by the institutional review board of Saitama Medical University since this study used anonymized data.

A detailed flow diagram of sample selection for analysis is shown in Fig. 1. We included clinical pregnancies after frozen-thawed single ET cycles between 2014 and 2017. Among 705,498 registered cycles, we excluded cancellation cycles $(n=8874)$, ET cycles with embryos transferred on days other than early cleavage and blastocyst stage $(n=19,116)$, cycles using frozen oocytes based on medical indication, such as fertility preservation for cancer patients $(n=35)$, other or unknown endometrial preparation protocols $(n=135,330)$, and double or multiple frozen-thawed ET cycles $(n=105,843)$. In the remaining 436,300 single ET cycles, 153,354 clinical pregnancies with known pregnancy outcomes were reported (clinical pregnancy rate $=35.1 \%$ ), and EP was reported in 792 of these cases $(0.52 \%)$.

Endometrial preparation protocols consisted of natural cycle (i.e., unstimulated), HRC, ovarian stimulation using CC, letrozole and exogeneous Gn. Both endometrial preparation protocols using CC and letrozole included cycles with exogeneous $\mathrm{Gn}$. We also used infertility diagnosis, embryo stage at transfer (early cleavage or blastocyst), assisted hatching and luteal phase support from the registry.

The definition of clinical pregnancy in the registry was the confirmation of a uterine gestational sac. The definition of EP was the confirmation of a gestational sac outside the uterine cavity (visualized by ultrasound) and also included clinically diagnosed cases in each ART facility, such as the combination of elevated serum human chorionic gonadotropin (hCG) levels without detection of a gestational sac. Heterotopic pregnancy, defined as an intrauterine pregnancy co-existing with an extrauterine gestational sac, was reported in 3 cases. In this study, heterotopic cases were defined as positive for the outcome, although the etiology for the occurrence of heterotopic pregnancy following single ET was unknown.

Statistical analysis. We first compared baseline characteristics and treatment information according to EP and non-EP groups using the chi-squared or Student's t-test. Odds ratios (ORs) and 95\% confidence intervals ( $95 \%$ CIs) of ART treatment, including endometrial preparation protocols, embryo stage at transfer and assisted hatching for EP, were calculated using generalized estimating equations with robust variance estimation adjusting for correlations within ART facilities. Confounders adjusted for the analysis were maternal age (categorized into 5-year age groups), tubal factor infertility diagnosis, male factor infertility, endometriosis and the year of registered cycles, following previously reported associations ${ }^{12}$. Furthermore, we adjusted for the diagnosis of other or unexplained causes of infertility because of the significant associations between these variables and incidence of EP (Table 1). Furthermore, we conducted several subgroup analyses as follows using generalized estimating equations with robust variance estimation adjusting for correlations within ART facilities; (1) with or without tubal factor infertility, (2) excluding both tubal factor infertility and endometriosis, (3) early cleavage and blastocyst ETs. The statistical adjustment was conducted for the same variables described above other than excluded/stratified variables for the subgroups analysis. All analyses were conducted using the STATA MP statistical package, version 16.1 (Stata, College Station, TX, USA). A two-tailed valued of $P<0.05$ was considered statistically significant.

\section{Data availability}

The datasets analyzed during the current study are not publicly available since the datasets include special carerequired personal information but are available from the corresponding author on reasonable request. 
Received: 25 December 2020; Accepted: 13 August 2021

Published online: 31 August 2021

\section{References}

1. Londra, L., Moreau, C., Strobino, D., Bhasin, A. \& Zhao, Y. Is the type of gonadotropin-releasing hormone suppression protocol for ovarian hyperstimulation associated with ectopic pregnancy in fresh autologous cycles for in vitro fertilization?. Fertil. Steril. 106, 666-672. https://doi.org/10.1016/j.fertnstert.2016.05.019 (2016).

2. Strandell, A., Thorburn, J. \& Hamberger, L. Risk factors for ectopic pregnancy in assisted reproduction. Fertil. Steril. 71, $282-286$. https://doi.org/10.1016/s0015-0282(98)00441-5 (1999).

3. Clayton, H. B. et al. Ectopic pregnancy risk with assisted reproductive technology procedures. Obstet. Gynecol. 107, 595-604. https://doi.org/10.1097/01.AOG.0000196503.78126.62 (2006).

4. Van Den Eeden, S. K., Shan, J., Bruce, C. \& Glasser, M. Ectopic pregnancy rate and treatment utilization in a large managed care organization. Obstet. Gynecol. 105, 1052-1057. https://doi.org/10.1097/01.AOG.0000158860.26939.2d (2005).

5. Hoover, K. W., Tao, G. \& Kent, C. K. Trends in the diagnosis and treatment of ectopic pregnancy in the United States. Obstet. Gynecol. 115, 495-502. https://doi.org/10.1097/AOG.0b013e3181d0c328 (2010).

6. Stulberg, D. B., Cain, L. R., Dahlquist, I. \& Lauderdale, D. S. Ectopic pregnancy rates and racial disparities in the Medicaid population, 2004-2008. Fertil. Steril. 102, 1671-1676. https://doi.org/10.1016/j.fertnstert.2014.08.031 (2014).

7. Perkins, K. M., Boulet, S. L., Kissin, D. M., Jamieson, D. J. \& National ART Surveillance (NASS) Group. Risk of ectopic pregnancy associated with assisted reproductive technology in the United States, 2001-2011. Obstet. Gynecol. 125, 70-78. https://doi.org/10. 1097/AOG.0000000000000584 (2015).

8. Jun, S. H. \& Milki, A. A. Assisted hatching is associated with a higher ectopic pregnancy rate. Fertil. Steril. 81, 1701-1703. https:// doi.org/10.1016/j.fertnstert.2003.10.042 (2004).

9. Zhang, B. et al. Reduced ectopic pregnancy rate on day 5 embryo transfer compared with day 3: A meta-analysis. PLoS ONE 12, e0169837. https://doi.org/10.1371/journal.pone.0169837 (2017).

10. Bouyer, J. et al. Risk factors for ectopic pregnancy: A comprehensive analysis based on a large case-control, population-based study in France. Am. J. Epidemiol. 157, 185-194. https://doi.org/10.1093/aje/kwf190 (2003).

11. Li, C. et al. Risk factors for ectopic pregnancy: A multi-center case-control study. BMC Pregnancy Childbirth 15, 187. https://doi. org/10.1186/s12884-015-0613-1 (2015).

12. Liu, H., Zhang, J., Wang, B. \& Kuang, Y. Effect of endometrial thickness on ectopic pregnancy in frozen embryo transfer cycles: An analysis including 17,244 pregnancy cycles. Fertil. Steril. 113,131-139. https://doi.org/10.1016/j.fertnstert.2019.09.003 (2020).

13. Yong, P. J. et al. Endometriosis and ectopic pregnancy: A meta-analysis. J. Minim. Invasive Gynecol. 27, 352-361 e352. https://doi. org/10.1016/j.jmig.2019.09.778 (2020).

14. Fang, C., Huang, R., Wei, L. N. \& Jia, L. Frozen-thawed day 5 blastocyst transfer is associated with a lower risk of ectopic pregnancy than day 3 transfer and fresh transfer. Fertil. Steril. 103, 655-661 e653. https://doi.org/10.1016/j.fertnstert.2014.11.023 (2015).

15. Huang, B. et al. Is frozen embryo transfer cycle associated with a significantly lower incidence of ectopic pregnancy? An analysis of more than 30,000 cycles. Fertil. Steril. 102, 1345-1349. https://doi.org/10.1016/j.fertnstert.2014.07.1245 (2014).

16. Ishihara, O., Kuwahara, A. \& Saitoh, H. Frozen-thawed blastocyst transfer reduces ectopic pregnancy risk: An analysis of single embryo transfer cycles in Japan. Fertil. Steril. 95, 1966-1969. https://doi.org/10.1016/j.fertnstert.2011.02.015 (2011).

17. Acharya, K. S. et al. Ectopic pregnancy rate increases with the number of retrieved oocytes in autologous in vitro fertilization with non-tubal infertility but not donor/recipient cycles: An analysis of 109,140 clinical pregnancies from the Society for Assisted Reproductive Technology registry. Fertil. Steril. 104, 873-878. https://doi.org/10.1016/j.fertnstert.2015.06.025 (2015).

18. Cai, J. et al. Low body mass index is associated with ectopic pregnancy following assisted reproductive techniques: A retrospective study. BJOG https://doi.org/10.1111/1471-0528.16378 (2020).

19. Levi Setti, P. E. et al. Seven years of vitrified blastocyst transfers: Comparison of 3 preparation protocols at a single ART center. Front. Endocrinol. (Lausanne) 11, 346. https://doi.org/10.3389/fendo.2020.00346 (2020).

20. Babayev, E. \& Robins, J. C. Endometrial thickness as an independent risk factor for ectopic pregnancy in in vitro fertilization: Should we change our counseling and practice?. Fertil. Steril. 113, 78-79. https://doi.org/10.1016/j.fertnstert.2019.10.033 (2020).

21. Ishihara, O. et al. Assisted reproductive technology in Japan: A summary report for 2016 by the Ethics Committee of the Japan Society of Obstetrics and Gynecology. Reprod. Med. Biol. 18, 7-16. https://doi.org/10.1002/rmb2.12258 (2019).

22. Jwa, S. C. et al. Ovarian stimulation increases the risk of ectopic pregnancy for fresh embryo transfers: An analysis of 68,851 clinical pregnancies from the Japanese Assisted Reproductive Technology registry. Fertil. Steril. https://doi.org/10.1016/j.fertnstert.2020. $06.032(2020)$.

23. Zeng, M. F. \& Li, L. M. Frozen blastocyst transfer reduces incidence of ectopic pregnancy compared with fresh blastocyst transfer: A meta-analysis. Gynecol. Endocrinol. 35, 93-99. https://doi.org/10.1080/09513590.2018.1497154 (2019).

24. Milki, A. A. \& Jun, S. H. Ectopic pregnancy rates with day 3 versus day 5 embryo transfer: A retrospective analysis. BMC Pregnancy Childbirth 3, 7. https://doi.org/10.1186/1471-2393-3-7 (2003).

25. Fanchin, R. et al. Uterine contractility decreases at the time of blastocyst transfers. Hum. Reprod. 16, 1115-1119. https://doi.org/ 10.1093/humrep/16.6.1115 (2001).

26. Schoolcraft, W. B., Surrey, E. S. \& Gardner, D. K. Embryo transfer: Techniques and variables affecting success. Fertil. Steril. 76, 863-870. https://doi.org/10.1016/s0015-0282(01)02731-5 (2001).

27. Groenewoud, E. R., Cantineau, A. E., Kollen, B. J., Macklon, N. S. \& Cohlen, B. J. What is the optimal means of preparing the endometrium in frozen-thawed embryo transfer cycles? A systematic review and meta-analysis. Hum. Reprod. Update 19, 458-470. https://doi.org/10.1093/humupd/dmt030 (2013).

28. Groenewoud, E. R. et al. A randomized controlled, non-inferiority trial of modified natural versus artificial cycle for cryo-thawed embryo transfer. Hum. Reprod. 31, 1483-1492. https://doi.org/10.1093/humrep/dew120 (2016).

29. Ghobara, T., Gelbaya, T. A. \& Ayeleke, R. O. Cycle regimens for frozen-thawed embryo transfer. Cochrane Database Syst. Rev. 7, CD003414. https://doi.org/10.1002/14651858.CD003414.pub3 (2017).

30. Boynukalin, F. B. et al. Frozen embryo transfers can reduce the risk of ectopic pregnancy in patients with non-tubal factor infertility undergoing elective freeze-all procedures. J. Reprod. Biol. Endocrinol. 4, 12-15 (2020).

31. Nakamura, Y. et al. Effects of clomiphene citrate on the endometrial thickness and echogenic pattern of the endometrium. Fertil. Steril. 67, 256-260. https://doi.org/10.1016/S0015-0282(97)81907-3 (1997).

32. Palomba, S. et al. Uterine effects of clomiphene citrate in women with polycystic ovary syndrome: A prospective controlled study. Hum. Reprod. 21, 2823-2829. https://doi.org/10.1093/humrep/del267 (2006).

33. Shao, R. et al. Clomiphene citrate causes aberrant tubal apoptosis and estrogen receptor activation in rat fallopian tube: Implications for tubal ectopic pregnancy. Biol. Reprod. 80, 1262-1271. https://doi.org/10.1095/biolreprod.108.074237 (2009).

34. Ministry of Internal Affairs and Communications Statistics Bureau. World Statistics 2018 [In Japanese]. https://www.stat.go.jp/ data/sekai/pdf/2018al.pdf (2018). 


\section{Acknowledgements}

This study was supported by Health and Labour Sciences Research Grants (H30-Sukoyaka-Ippan-002 to SCJ, AK and OI) and AMED (JP21gk0110046 to OI). The authors thank the JSOG for providing the data and all participating clinics in the Japanese ART registry for their support in data collection. We thank Charles Allan, $\mathrm{PhD}$, from Edanz Group (https://en-author-services.edanzgroup.com/ac) for editing a draft of this manuscript.

\section{Author contributions}

S.C.J. conceived the study design. A.K. and O.I. collected data for the analysis. S.C.J. analyzed the data, and M.T., A.K., T.K., and O.I. interpreted the results. S.C.J. wrote the first draft of the manuscript and M.T., A.K., T.K., and O.I. finalized the draft. All authors were involved in writing the paper and had final approval of the submitted and published versions.

\section{Competing interests}

The authors declare no competing interests.

\section{Additional information}

Supplementary Information The online version contains supplementary material available at https://doi.org/ 10.1038/s41598-021-97044-6.

Correspondence and requests for materials should be addressed to S.C.J.

Reprints and permissions information is available at www.nature.com/reprints.

Publisher's note Springer Nature remains neutral with regard to jurisdictional claims in published maps and institutional affiliations.

(c) (i) Open Access This article is licensed under a Creative Commons Attribution 4.0 International License, which permits use, sharing, adaptation, distribution and reproduction in any medium or format, as long as you give appropriate credit to the original author(s) and the source, provide a link to the Creative Commons licence, and indicate if changes were made. The images or other third party material in this article are included in the article's Creative Commons licence, unless indicated otherwise in a credit line to the material. If material is not included in the article's Creative Commons licence and your intended use is not permitted by statutory regulation or exceeds the permitted use, you will need to obtain permission directly from the copyright holder. To view a copy of this licence, visit http://creativecommons.org/licenses/by/4.0/.

(c) The Author(s) 2021 